\title{
PERFORMANCE ANALYSIS OF SERPENTINE SPRINGS COMPLIANT TO OUT-OF-PLANE OSCILLATION
}

\author{
NGUyen VAn DuOng \\ International Training Institute for Materials Science, Hanoi University of Science and Technology, Hanoi, Vietnam, and \\ FTP University, Hanoi, Vietnam
}

NGuyen Quoc Chien

International Training Institute for Materials Science, Hanoi University of Science and Technology, Hanoi, Vietnam DANG VAN HIEU

FPT University, Hanoi, Vietnam

Chu Manh Hoang

International Training Institute for Materials Science, Hanoi University of Science and Technology, Hanoi, Vietnam

e-mail: hoangcm@itims.edu.vn; hoang.chumanh@hust.edu.vn

\begin{abstract}
The performance of two serpentine type springs is comparatively investigated. The first type is composed of straight beams and the second one is composed of circular arcs. Based on comparing calculation results and simulation data, the crab-leg spring model is appropriate for evaluating the stiffness of springs. To obtain the operation mode to be the first mode, the number of turns and the opening angle of springs should be increased. The performance of springs is evaluated via analysis of mode coupling. This study is useful for choosing an appropriate serpentine spring and the stiffness calculation model for applications in microelectroemchanical sensors and actuators.
\end{abstract}

Keywords: serpentine spring, out-of-plane oscillation, curved spring beam, straight spring beam, mode coupling

\section{Introduction}

The spring with a single straight beam has been applied in a variety of microelectroemchanical devices including flexural and torsional oscillations in sensors, actuators, RF MEMS switches, and others (Liu et al., 2007; Legtenberg et al., 1996; Huang et al., 2009; Sharaf and Sedky, 2012; Matsumoto et al., 1999; Peroulis et al., 2003; Nguyen et al., 2017; Su et al., 2005; Gu et al., 2006). However, for applications requiring large displacements and low spring constants, serpentine springs have been developed to fulfill these requirements (Hieu et al., 2020; Chou et al., 2016; Barillaro et al., 2005; Rouabah et al., 2005). In addition, using serpentine springs, the overall device area is also miniaturized. The serpentine springs have been designed for displacements in plane, torsional, and out-of-plane oscillations (Su et al., 2005; Chou et al., 2016; Barillaro et al., 2005; Rouabah et al., 2005). Furthermore, the designed spring is required to be compliant to the interested motion directions, while it has ability to resist to the other undesired motion directions (Hieu et al., 2020; Weinberg and Kourepenis, 2006). In these reports, the serpentine springs are almost composed of straight spring beams. There are not many studies on the serpentine springs with curved spring beams. Therefore, theoretical models for evaluating accurately the out-of-plane stiffness of these kinds of springs are necessary for the initial design phase. Moreover, comparing the performance of these two kinds of springs is useful for applications in designing microelectroemchanical devices. 
In this paper, we present models for evaluating the stiffness of serpentine springs consisting of straight and curved spring beams compliant to out-of-plane oscillations for developing microelectroemchanical sensors and actuators. We examine the accuracy of the established theoretical models in evaluating the stiffness of the two types of serpentine springs by comparing the calculation results with the simulation data. In addition, we also compare the performance of the two types of serpentine springs with the similar dimensions based on analyzing the mode coupling.

\section{Analytical model}

\subsection{Model of serpentine springs}

We design two similar spring structures. Both structures consist of a circular plate with radius $R_{p}$, which is suspended by two symmetrical serpentine springs, see Figs. 1a and 1b. The opening angle of springs is $\alpha$. The thickness of the center plate as well as the spring is $t$. The difference between the two types of springs is that one structure (Type 1) uses straight beam springs, while the other (Type 2) uses curved (circular arc) beam springs. To compare the performance of the two types of springs, the effective length of spring circular arcs (Type 2) is the same as that of straight spring beams (Type 1).

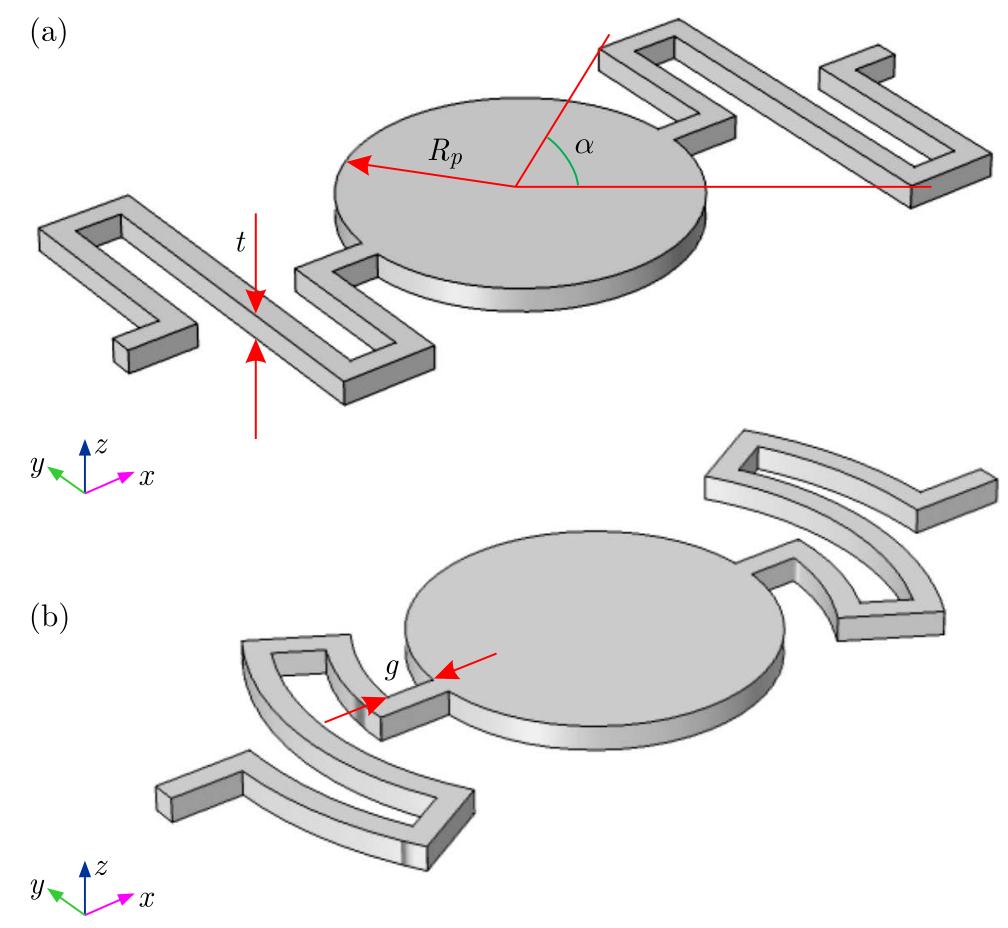

Fig. 1. Structure of serpentine springs with straight beams (a) and circular arcs (b); $g$ is the gap between spring circular arcs

In the following, theoretical models are established for evaluating the stiffness in the $z$-axis direction as well as the operation frequency of the two spring types. The accuracy of the calculated models is evaluated by comparing the calculated results with the simulation data.

\subsection{Calculation and simulation method}

The first calculation method is based on analyzing the serpentine spring with straight beams (Type 1), Fig. 1a, divided into basic crab-leg springs connected in series. Figure 2a shows the 3D structure of the serpentine spring. The serpentine spring is divided into basic crab-leg springs connected in series, Figs. 2b and 2c. 
(a)

(a)

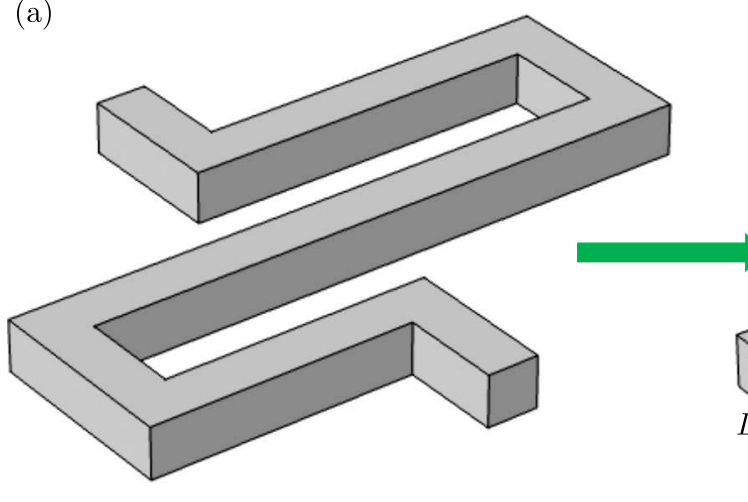

(b)

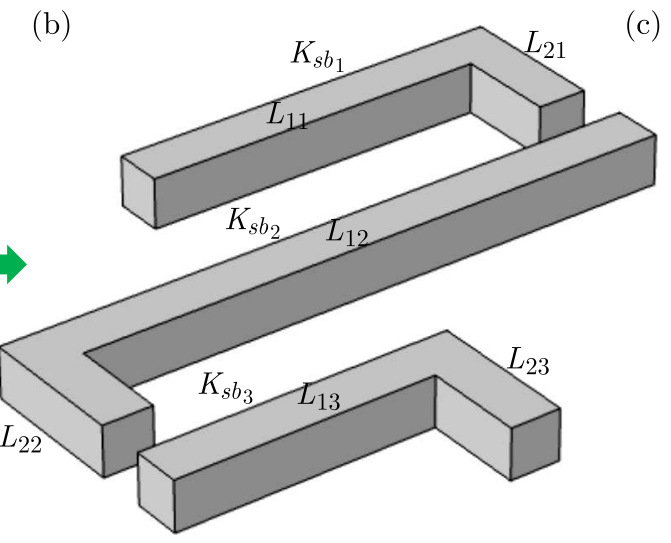

(c)

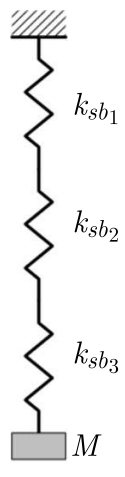
$y \stackrel{\Lambda}{z}^{z} x$

Fig. 2. 3D view of the serpentine spring with straight beams (a), serpentine spring divided into basic crab-leg springs connected in series (b), and equivalent series spring model (c)

The stiffness $k_{s b_{i}}$ of the $i$-th crab-leg spring with straight beams is given by (Hongwen, 2004; Lobontiu and Garcia, 2005)

$$
k_{s b_{i}}=\frac{1}{\frac{\left(L_{1 i}-K^{\prime}\right)^{2} L_{2 i}}{G I_{t}}+\frac{L_{1 i}^{3}+L_{2 i}^{3}+3 K^{\prime} L_{1 i}\left(K^{\prime}-L_{1 i}\right)}{E I_{y}}}
$$

where $L_{1 i}$ and $L_{2 i}$ are denoted in Fig. $2 \mathrm{~b}, G=E /[2(1+\gamma)]$ is the shear modulus, $E$ is Young's modulus of the spring material, which is $1.7 \cdot 10^{11} \mathrm{~Pa}$ for silicon material, $I_{y}=w t^{3} / 12$ is the inertial moment of the cross section, $I_{t}=w t^{3} / 3$ is the polar moment of inertia of the cross section, in which $w$ is width of the spring and $t$ is thickness of the spring. $K^{\prime}$ in Eq. (2.1) is calculated by

$$
K^{\prime}=\frac{\frac{L_{1 i}^{2}}{2 E I_{y}}+\frac{L_{1 i} L_{2 i}}{G I_{t}}}{\frac{L_{1 i}}{E I_{y}}+\frac{L_{2 i}}{G I_{t}}}
$$

In Eq. (2.1), $L_{1 i}=g$ and $L_{2 i}=2 R \tan \alpha$, here $R=R_{p}+n g+(n-1) w ; i$ shows the $i$-th spring straight beam.

To calculate the total stiffness $K_{t s b}$ of the serpentine spring with straight beams, the principle of springs connected in series is used

$$
K_{t s b}=\frac{2}{\sum_{i=1}^{n} \frac{1}{k_{s b_{i}}}}
$$

For Type 2 in Fig. 1b, we can also analyze the spring divided into basic circular arc springs connected in series as shown in Fig. 3.

The stiffness of the circular arc spring $k_{c b_{i}}$ is given by (Hongwen, 2004; Lobontiu and Garcia, 2005)

$$
k_{c b_{i}}=\frac{4}{R^{3}\left(\frac{2 \alpha-\sin 2 \alpha}{G I_{t}}+\frac{6 \alpha-8 \sin \alpha+\sin 2 \alpha}{E I_{y}}\right)}
$$

where

$$
R=R_{p}+i g+(i-1) w
$$


(a)

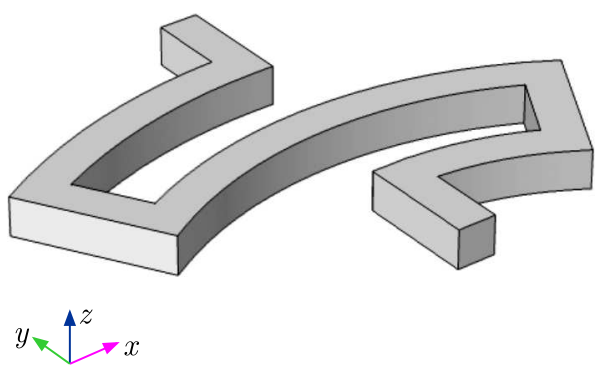

(b)

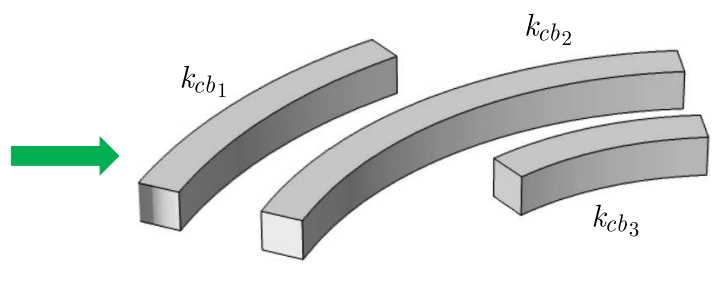

(c)

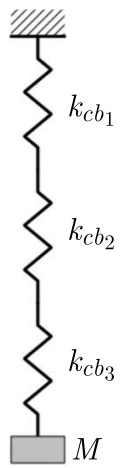

Fig. 3. 3D view of the serpentine spring with circular arcs (a), serpentine spring divided into basic circular arc springs connected in series (b), and the equivalent series spring model (c)

$i$ shows the $i$-th spring circular arc. Therefore, the total stiffness $K_{t c b}$ of the serpentine spring with spring circular arcs, the principle of springs connected in series is used

$$
K_{t c b}=\frac{2}{\sum_{i=1}^{n} \frac{1}{k_{c b_{i}}}}
$$

The $z$-axis natural frequency of the spring system suspending the center plate can be calculated by (Hieu et al., 2020)

$$
f=\frac{1}{2 \pi} \sqrt{\frac{K}{m}}
$$

where

$$
m=\rho V_{p}+\frac{13}{35} \rho V_{s}
$$

In Eq. (2.8), $V_{p}=\pi R_{p}^{2} t$ is volume of the plate, $V_{s}=V_{b}+V_{g}$ is volume of the serpentine springs, in which for the straight beam: $V_{b}=L_{2} w t$ and $V_{g}=g w t$; for the circular arc: $V_{b}=\pi\left[(R+w)^{2}-R^{2}\right][\alpha /(2 \pi)]$ and $V_{g}=g w t$, and $\rho$ is density of the spring material; for the silicon material $\rho=2329 \mathrm{~kg} / \mathrm{m}^{3}$.

Thus, we have built models for evaluating the out-of-plane stiffness of the two spring types. In the following, we will present results obtained by employing the above built equations. The calculation results are compared to those obtained from numerical simulation using Comsol multiphysics 4.3. In this study, the used Comsol module is the MEMS module. We use triangular mesh elements to divide the spring structure for analyzing the modes.

\section{Results and discussion}

In this study, the interested operation mode of the two types of springs is the out-of-plane oscillation mode in the $z$-axis direction (mode- $z$ ). As a representation, Fig. 4 shows the first three modes simulated, respectively, for the serpentine spring with straight beams, Figs. 4a-c for the serpentine spring with circular arcs, Figs. 4d-f, for the number of turns of the spring $n=3$. Thus, the first mode is the interested mode- $z$ (Figs. $4 \mathrm{a}$ and $4 \mathrm{~d}$ ). The second mode is the in-plane oscillation mode along the $x$-axis due to flexural vibration of the spring beams, which is called to be mode- $x$ (Figs. $4 \mathrm{~b}$ and $4 \mathrm{e}$ ). The third mode is the torsional mode (mode-tor) around the $x$-axis (Figs. 4c and 4f). The natural frequencies of the first three modes for Type 1 and 2 are also shown in Figs. 4a-f, respectively. The geometry parameters used in this simulation are 
(a)

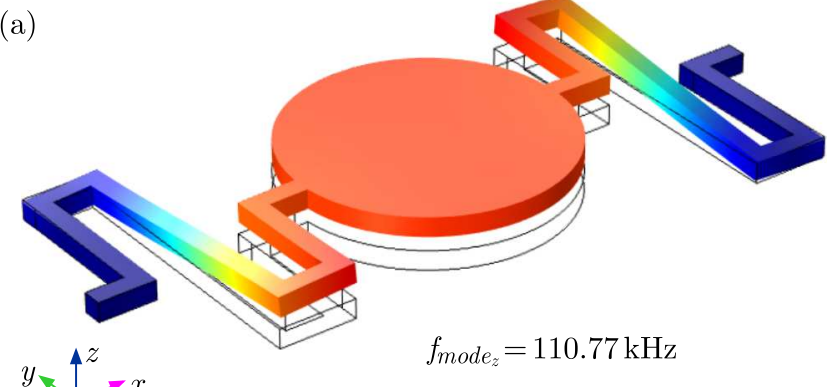

(b)

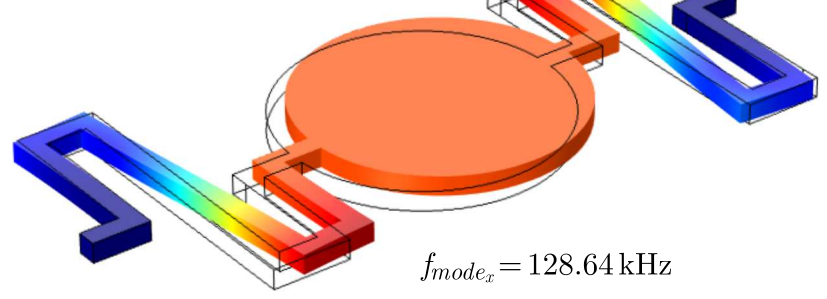

(d)

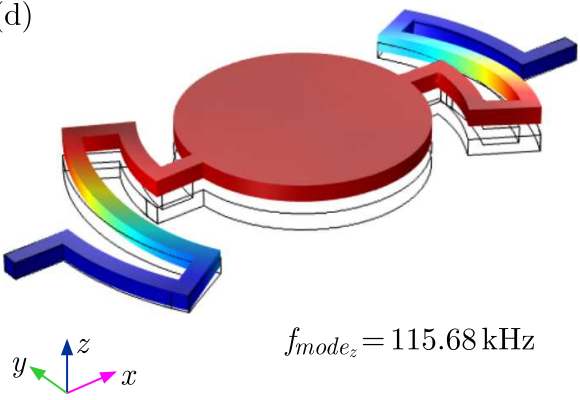

(e)

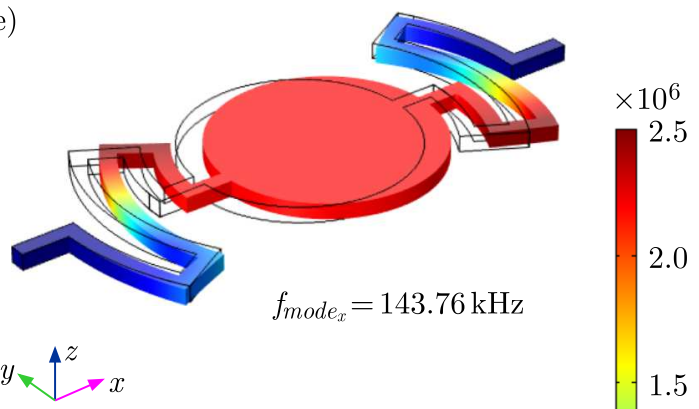

(f)

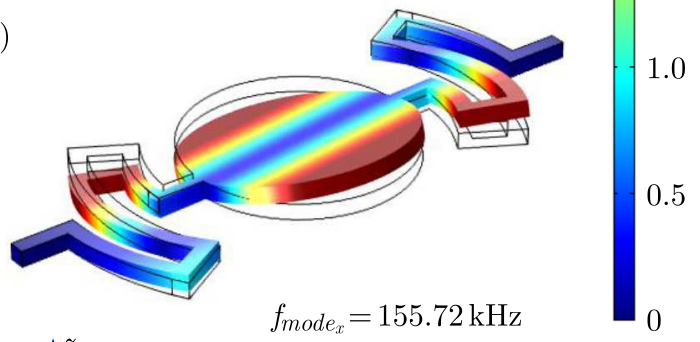

$y \stackrel{4}{\sim} \times x$ (c)

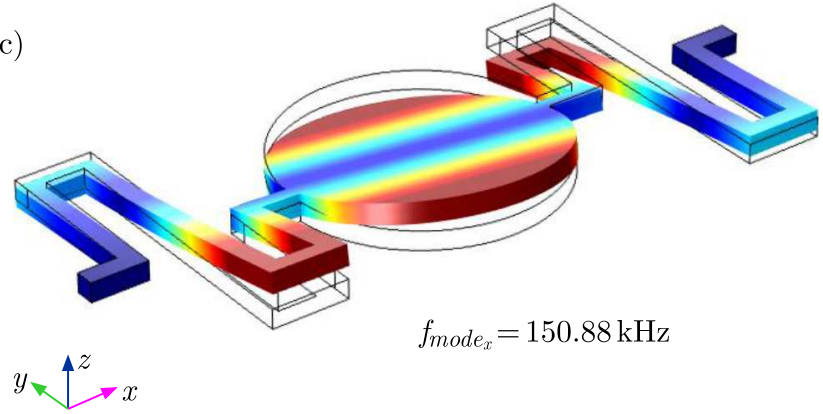

Fig. 4. The first three modes simulated respectively for: the serpentine spring with straight spring beams (a)-(c) and the serpentine spring with circular $\operatorname{arcs}(d)-(f)$

as follows: radius of the center plate $R_{p}=70 \mu \mathrm{m}$, gap between the beams $g=20 \mu \mathrm{m}$, width $w=10 \mu \mathrm{m}$ and thickness $t=10 \mu \mathrm{m}$, and the opening angle $\alpha=60^{\circ}$.

In the following, we will calculate the stiffnesses as well as frequencies of the two types of springs using the theoretical models built in Section 2 and compare these calculation results with respective simulation data. For convenience in presentation, we denote the results as follows: $f_{s s b_{i}}$ and $f_{s c b_{i}}$ are the frequency of the serpentine spring structure with the straight beams and the circular arcs using simulation, respectively; $f_{c c l m_{i}}$ is the frequency of the serpentine spring structure calculated by the crab-leg method and $f_{c c s m_{i}}$ is the frequency of the serpentine spring structure calculated by the circular arc spring method; $\Delta f_{s_{i}}$ is the difference between the frequency of the straight beam spring using simulation $f_{s s b_{i}}$ and that calculated by the crab-leg method $f_{c c l m}$; and $\Delta f_{c_{i}}$ is the difference between the frequency of the circular arc spring found from simulation $f_{s c b_{i}}$ and that calculated by the circular arc spring method $f_{c c s m_{i}}$. Here, the difference between the calculation and simulation results is in percent, for example, $\Delta f_{s_{i}}=\left(f_{c c l m_{i}}-f_{s s b_{i}}\right) / f_{s s b_{i}} x \cdot 100 \%$. Here, $i=1,2,3$ corresponds to the order number of circular arcs/straight beams.

In the first case, we consider the springs with $n=1$. The calculated and simulated frequencies of the springs in the $z$-axis direction as a function of width $w$, thickness $t$, and the opening angle $\alpha$ are shown in Figs. 5a, 5b and 5c, respectively. In the calculation and simulation, we vary the interested dimension parameter while fixing the remaining dimension parameters. 
(a)

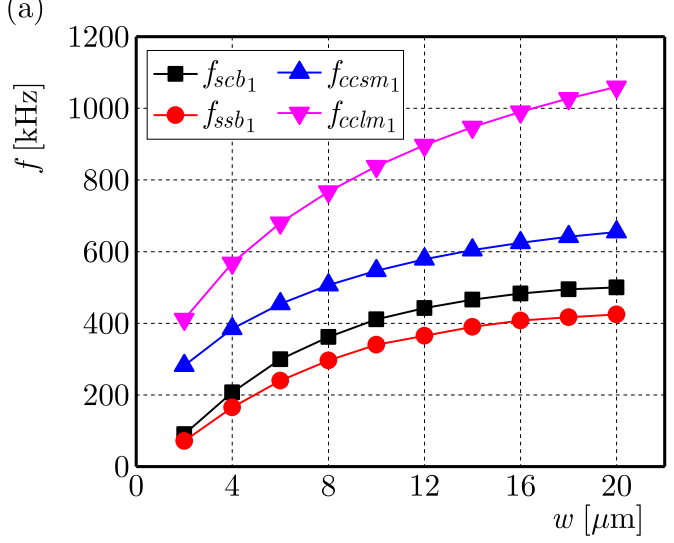

(b)

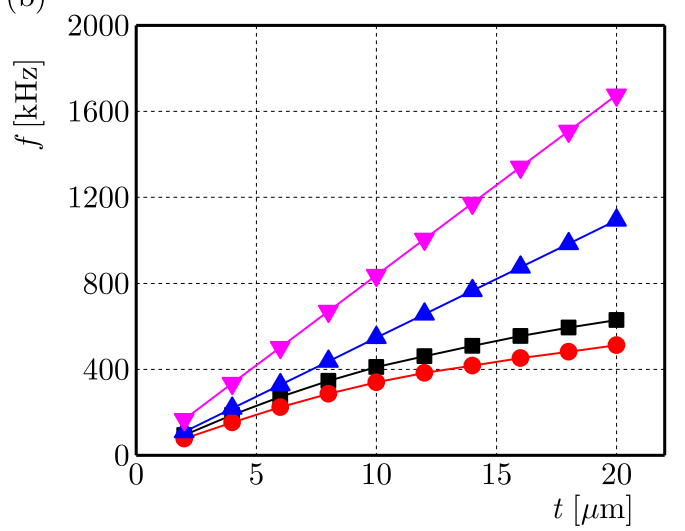

(c)

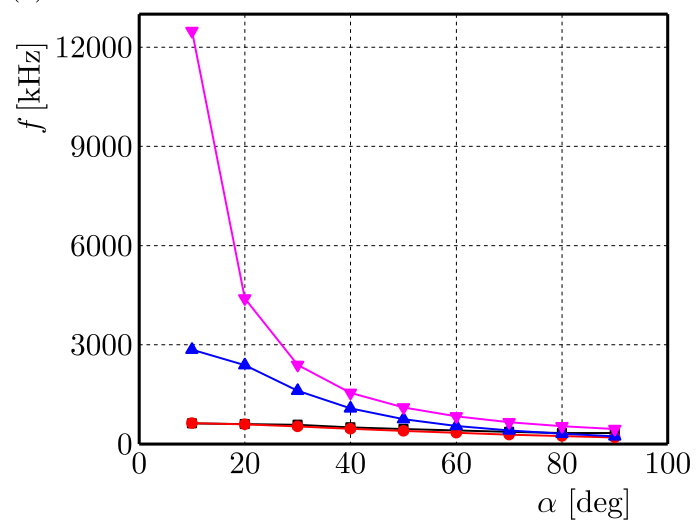

(d)

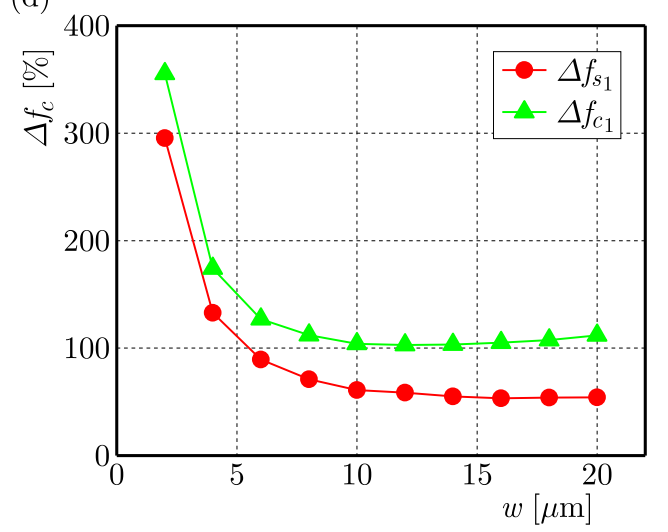

(e)

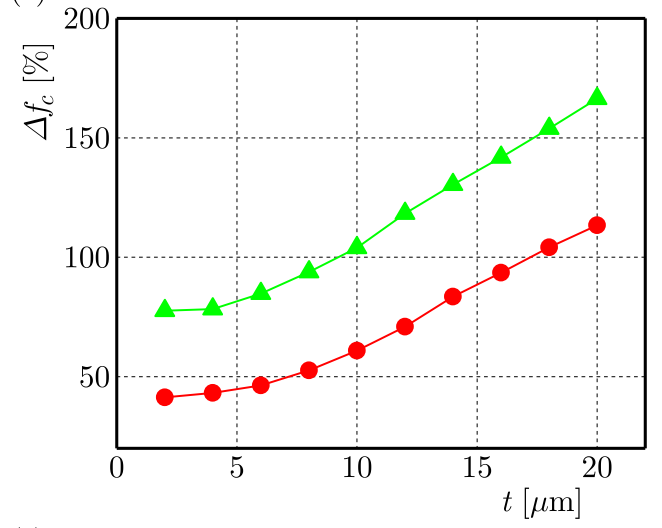

(f)

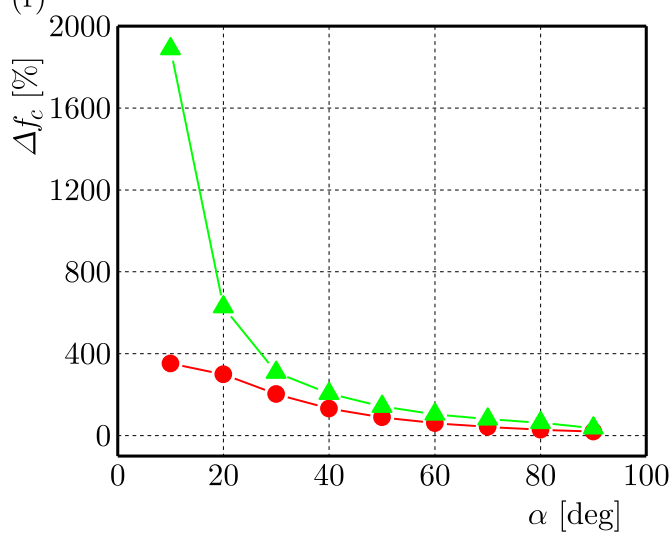

Fig. 5. The operation frequency of two spring types with $n=1$ investigated by both theoretical calculation and simulation as a function of $w(\mathrm{a}), t(\mathrm{~b})$ and $\alpha(\mathrm{c})$. The difference between the calculated and simulated results investigated as a function of $w(\mathrm{~d}), t(\mathrm{e})$, and $\alpha$ (f)

When the width $w$ and the thickness $t$ varies from 2 to $20 \mu \mathrm{m}$, the frequencies of the springs increase monotonically with $w$ (Fig. $5 \mathrm{a}$, in this case, $t$ and $\alpha$ are fixed at $10 \mu \mathrm{m}$ and $60^{\circ}$, respectively) and $t$ (Fig. 5b, in this case, $w$ and $\alpha$ are fixed at $10 \mu \mathrm{m}$ and $60^{\circ}$, respectively). In general, the frequency depends quite linearly on $t$, while it depends on $w$ having the shape of square of $w$. These relations agrees with the frequency expression on $w$ and $t$, Eq. (2.7). Especially, when width $w$ varies from 2 to $20 \mu \mathrm{m}$, the frequency curves tend to approach asymptotic values. In this investigated range of $w$, the frequency of oscillation along the axis $f_{c c l m_{1}}$ is varied from $67 \mathrm{kHz}($ at $w=2 \mu \mathrm{m})$ to $400 \mathrm{kHz}($ at $w=14 \mu \mathrm{m})$, Fig. 5a. $f_{c c l m_{1}}$ also appreaches more quickly the asymptotic value than $f_{s c b_{1}}, f_{s s b_{1}}$ and $f_{c c s m_{1}}$. As the thickness $t$ is varied in the range of $2-20 \mu \mathrm{m}$, the simulation results of $f_{s c b_{1}}$ and $f_{s s b_{1}}$ show a nonlinear relation, while the calculated frequencies $f_{c c l m_{1}}$ and $f_{c c s m_{1}}$ are quite linear lines (Fig. 5b). All the frequency values significantly 
increase with $t$, from $77 \mathrm{kHz}$ to $512 \mathrm{kHz}\left(f_{\text {ccsm }}\right)$, when $t$ is varied from 2 to $20 \mu \mathrm{m}$. Figure $5 \mathrm{~d}$ shows that when the width $w$ increases from 2 to $20 \mu \mathrm{m}$, the error decreases, in contrast, when the thickness $t$ increases from 2 to $20 \mu \mathrm{m}, \Delta f_{s_{1}}$ and $\Delta f_{c_{1}}$ increase, see Fig. 5e. Thus, $\Delta f_{s_{1}}$ and $\Delta f_{c_{1}}$ are both greater than $40 \%$ for $n=1$.

When $\alpha$ is varied from $10^{\circ}$ to $90^{\circ}$ while $w$ and $t$ are both fixed at $10 \mu \mathrm{m}$, the theoretically calculated and simulated $z$-axis frequencies strongly decrease with $\alpha$, especially, those obtained from calculations of $f_{c c l m_{1}}$ and $f_{c c s m_{1}}$. This is explained that when $\alpha$ increases, the effective length of the springs increases correspondingly. It is clear that the simulated $z$-axis frequencies of the serpentine spring with straight beams $f_{s c b_{1}}$ are the same as those of the serpentine spring with circular arcs $f_{s s b_{1}}$, Fig. 5c. When $\alpha$ in the range $60^{\circ}$ to $90^{\circ}$, the calculated results are in good agreement with those obtained from the simulation data. In this investigated range of $\alpha$, the $z$-axis frequency $f_{s c b_{1}}$ is modified from $20 \mathrm{kHz}\left(\right.$ for $\alpha=90^{\circ}$ ) to $630 \mathrm{kHz}$ (for $\alpha=10^{\circ}$ ). Figure 5 f shows that when $\alpha$ increases from $10^{\circ}$ to $90^{\circ}$, the calculation errors decrease, when $\alpha=90^{\circ} \Delta f_{s_{1}}$ and $\Delta f_{c_{1}}$ are $18 \%$ and $27 \%$, respectively. In general, the calculated results by the crab-leg method are closer to the simulation data than those calculated by the method using the spring circular arcs, Figs. 5d-f.

(a)

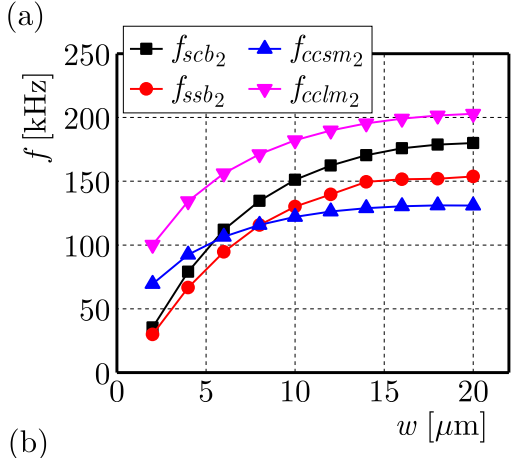

(b)

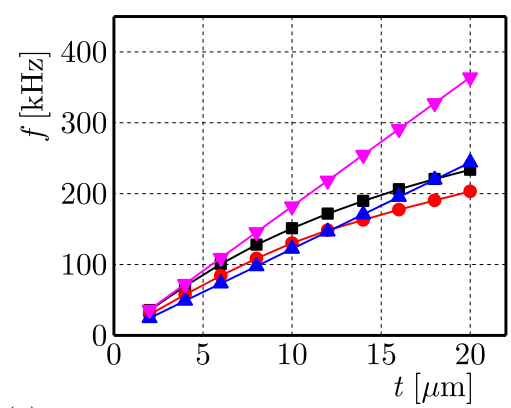

(c)

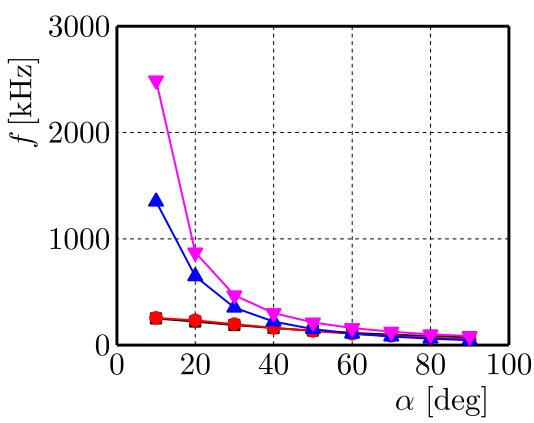

(d)

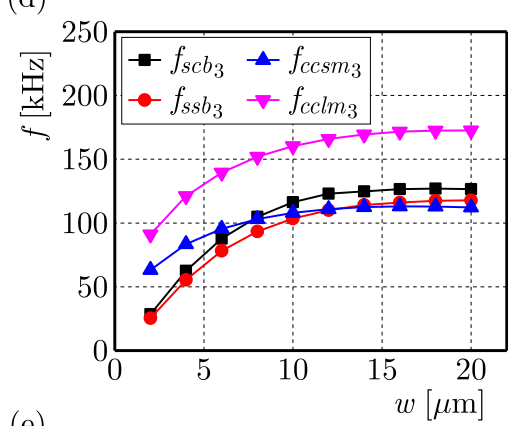

(e)

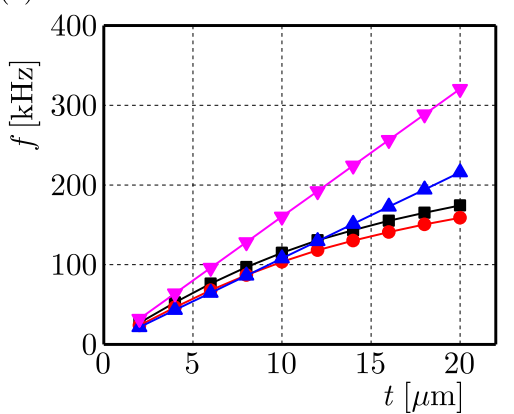

(f)

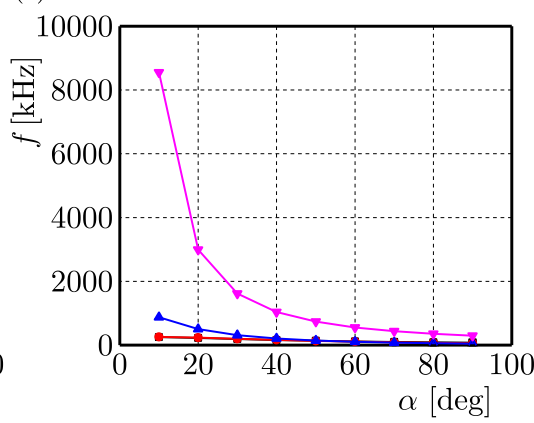

(g)

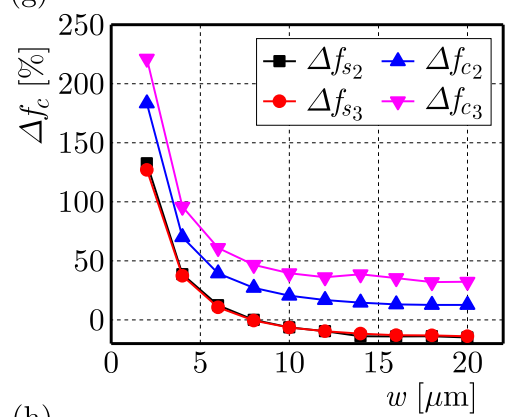

(h)

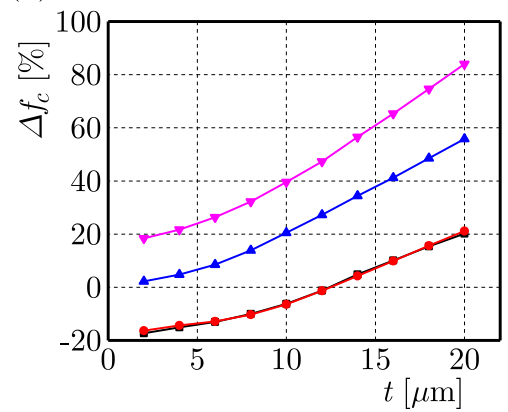

(i)

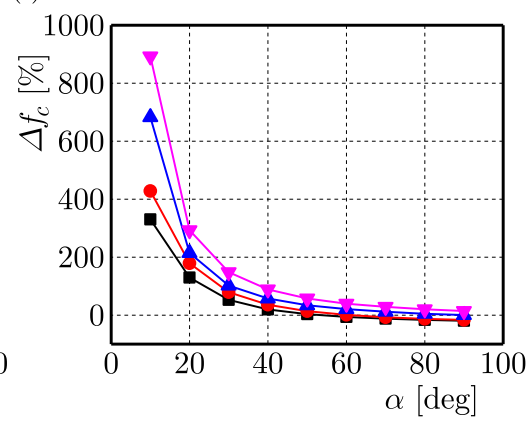

Fig. 6. The operation frequency of the two spring types investigated by both theoretical calculation and simulation as a function of $w, t$, and $\alpha$ for $n=2$ (a)-(c) and $n=3$ (d)-(f). The difference between the frequency of calculation and simulation depends on a function of dimensional parameters $w(\mathrm{~g})$, $t(\mathrm{~h})$, and $\alpha$ (i) 
The results of calculation and simulation while changing the same dimensional parameters of the serpentine springs with $n=2$ and $n=3$ are shown in Figs. 6a-c and Figs. 6d-f, respectively.

When a dimensional parameter is varied, the remaining parameters are also fixed at the values the same as those in the case $n=1$. Similar to the case $n=1$, the relations between the $z$-axis frequencies on $w$ and $t$ reflect the theoretical depencence of the frequency on $w$ and $t$, Eq. (2.7). In the investigated ranges of $w, t$, and $\alpha$, the $z$-axis frequencies $f_{s c b}$ are varied from $30 \mathrm{kHz}$ to $358 \mathrm{kHz}$, for $n=2$, Figs. 6a-c, and from $25 \mathrm{kHz}$ to $251 \mathrm{kHz}$, for $n=3$, Figs. 6d-f. The difference between the frequency of calculation and simulation for $n=2$ and $n=3$ are shown in Figs. 6g-i. In general, the calculated results using the crab-leg spring method are in better agreement with the simulation data. In addition, the errors decrease when the number of spring straight beams/spring circular arcs increases. The errors between the calculated and simulated results are less than $20 \%$ for the investigated dimensional ranges, $w$ from $12-20 \mu \mathrm{m}$, $t$ from $2-8 \mu \mathrm{m}$, and $\alpha$ from $70^{\circ}-90^{\circ}$ for $n=2$, whereas for $n=3$, the errors are less than $20 \%$ for the investigated dimensional ranges, $w$ from $6-20 \mu \mathrm{m}, t$ from $2-18 \mu \mathrm{m}$, and $\alpha$ from $80^{\circ}-90^{\circ}$.

As introduced above, in this study, we design serpentine springs in which the effective length of circular arcs is similar to that of straight beams. However, using the two models for evaluating the frequency of the two spring types, the difference in frequency evaluation $\Delta f_{c}$ is shown in Figs. $7 \mathrm{a}$-c for $n=1,2,3$. It is clear that the stiffness is calculated by the crab-leg spring model to be more accurate than for the serpentine spring consisting of straight beams as well as equivalent circular arcs, Figs. 5 and 6. From Figs. 7a-c, when $n$ increases, the calculation difference of the two models is decreased. For values of $n \geqslant 2$, the calculation difference $\Delta f_{c}$ is almost independent of $n$. The results in Figs. 7a and b show that $\Delta f_{c}$ increases with $w$ while it is almost constant to $t$ for $n=1,2,3$. In particular, there are minima on the curves of $\Delta f_{c}(\alpha)$, Fig. 7c. When $n=1$, the minimum occurs at $\alpha=40^{\circ}$ while it occurs at $\alpha=25^{\circ}$ with $n \geqslant 2$. This means that at $\alpha=25^{\circ}$, the difference in frequency evaluation using the two models $\Delta f_{c}$ is the smallest (less than $33 \%)$.

To compare the performance of the two spring types, we evaluate the mode coupling by investigating the difference ( $\delta f$ in percent) between the operation mode frequency $\left(f_{s s b_{i}}, f_{c c s m_{i}}\right)$ and the undesired nearest mode frequency $\left(f_{s s b_{2 i}}, f_{c c s m_{2 i}}\right)$ for Type 1 and springs. In this comparison, we choose $n=3$ for investigation. The $\delta f$ values investigated as a function of $w, t$, and $\alpha$ are shown in Figs. 7d, 7e and 7f, respectively. It is clear that the performance of the two springs is almost the same, although the area of the device using the serpentine spring consisting of circular arcs is a bit miniaturized (reduced by 15\%) compared to that of the serpentine spring consisting of straight beams.

In applications, the operation mode having the lowest frequency (the first mode) is always preferred; however, the mode order depends on the representative dimensional parameters of springs. Although the previous researches have concentrated on individual investigation of the performance of two serpentine spring types, the dependence of the mode order on the representative dimensional parameters of springs, especially on $n$ and $\alpha$, is seldom studied. Here, we present investigation of the dependence of the mode order on $n$ and $\alpha$. The investigated results are shown in Fig. 8. The parameters of the springs are fixed the same as in Fig. 4, while $\alpha$ is varied. It is clear that when $n$ increases, the operation mode (mode- $z$ ) is transformed from the second mode (Figs. 8a and 8d) into the first mode, Figs. 8b and 8e, for $n=2$ and Figs. 8c and 8f for $n=3$. At $n=3$, the operation mode is the first mode for $\alpha$ varied from $10^{\circ}$ to $90^{\circ}$. Thus, to ensure the operation mode to be the first mode, $n$ and $\alpha$ should be modified in the increased trend. 
(a)

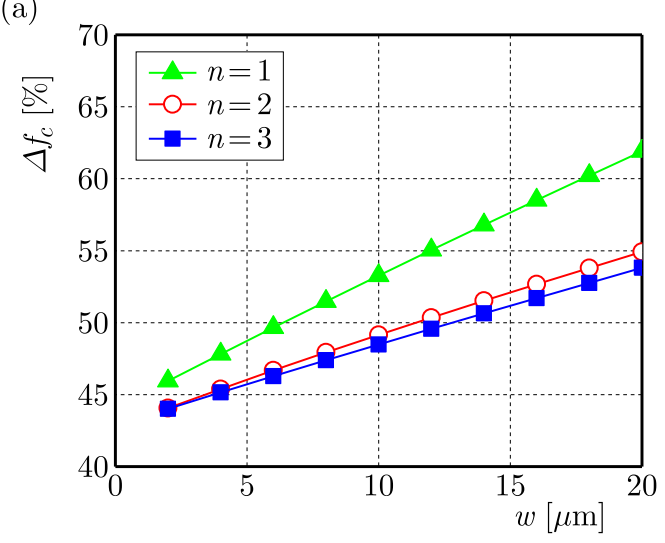

(b)

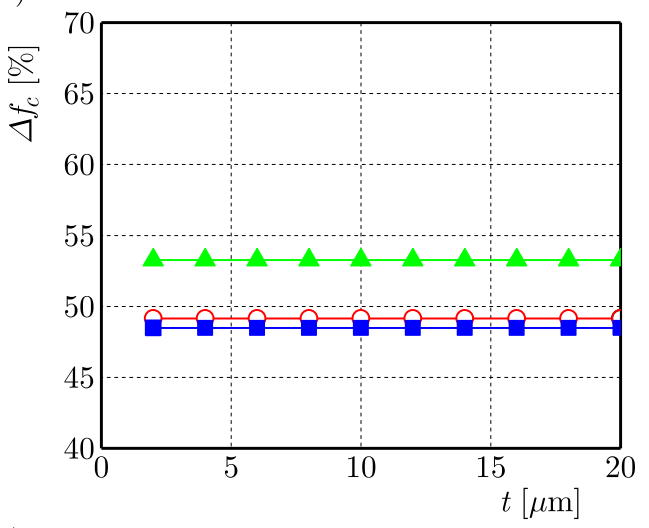

(c)

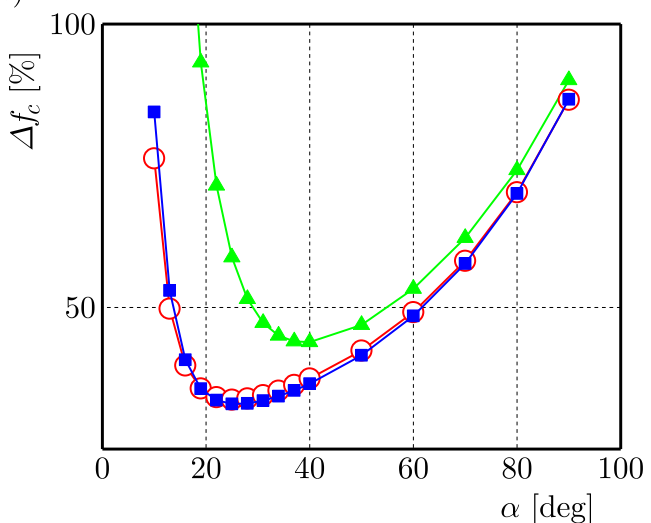

(d)

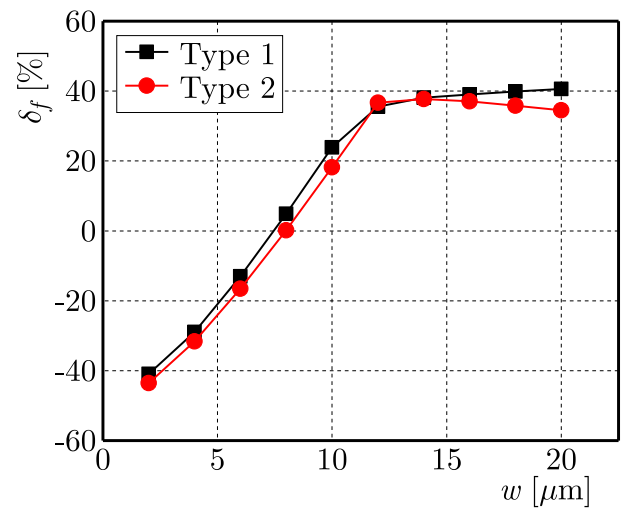

(e)

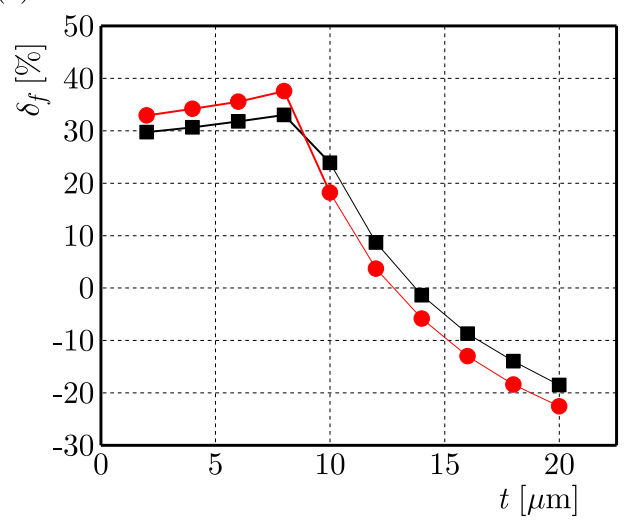

(f)

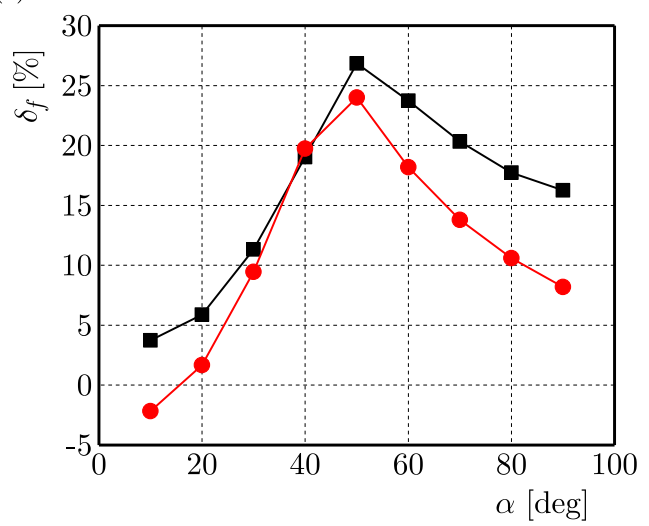

Fig. 7. $\Delta f_{c}$ calculated by the two models as a function of $w(\mathrm{a}), t(\mathrm{~b})$, and $\alpha$ (c) for $n=1,2,3$; $\delta f$ for the two spring types investigated as a function of $w(\mathrm{~d}), t(\mathrm{e})$, and $\alpha$ (f) for $n=3$

\section{Conclusion}

We have built and examined theoretical models for evaluating the stiffness of two types of equivalent serpentine springs. The first type is composed of straight beams and the second one is composed of circular arcs. When the number of straight beams/circular arcs increases, the theoretical models are more suitable for evaluating the stiffness of the serpentine springs. Based on comparing the calculation results and simulation data, the crab-leg spring method is appropriate for evaluating the stiffness of the two types of springs. There is good agreement between the calculation results and simulation data in wide ranges of the representative dimensions of the springs. The errors between the calculation and simulation results are less than $20 \%$ for the variation of spring dimensional parameters: width $w$ from $6-20 \mu \mathrm{m}$, thickness $t$ from $2-18 \mu \mathrm{m}$, 
(a)

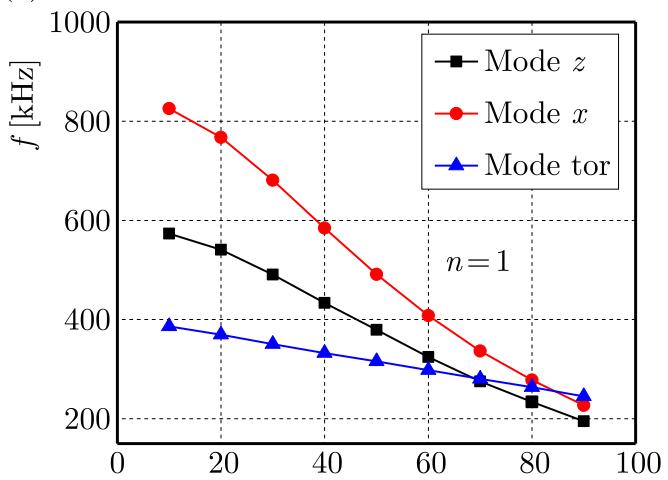

(b)

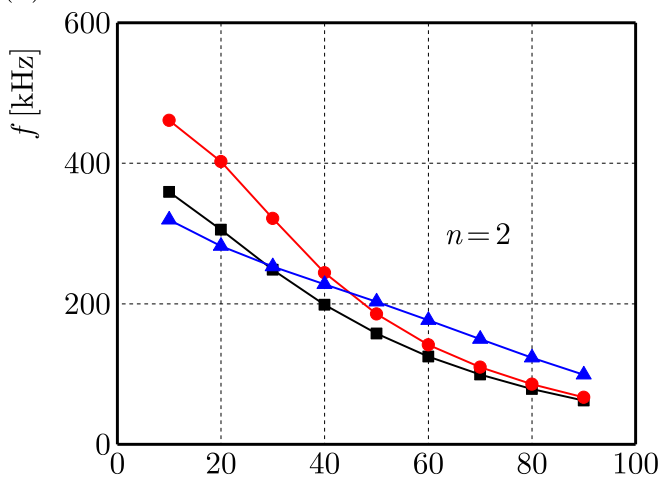

(c)

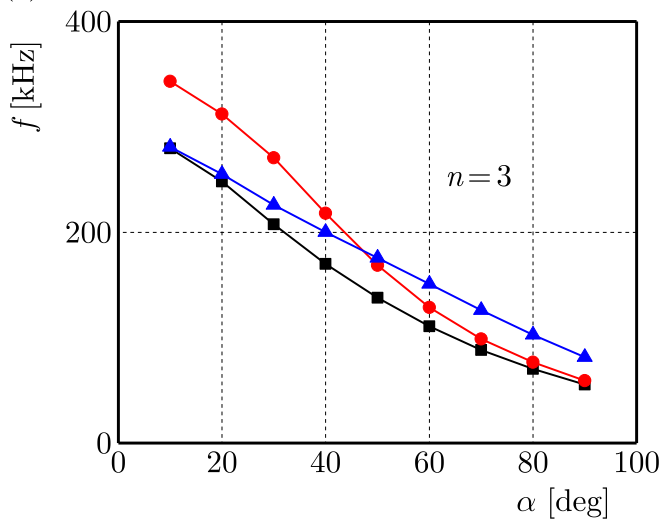

(d)

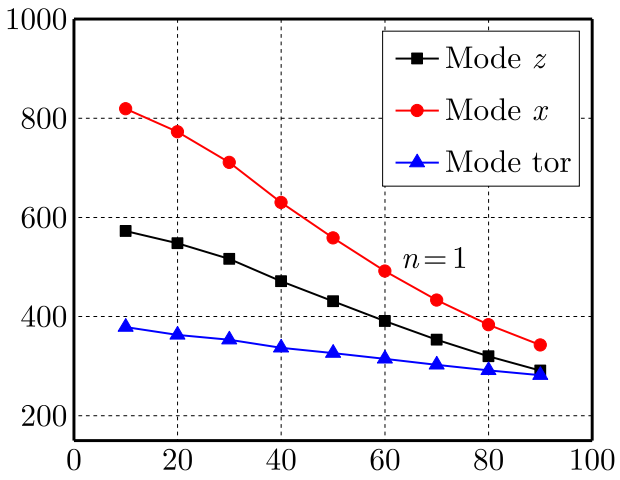

(e)

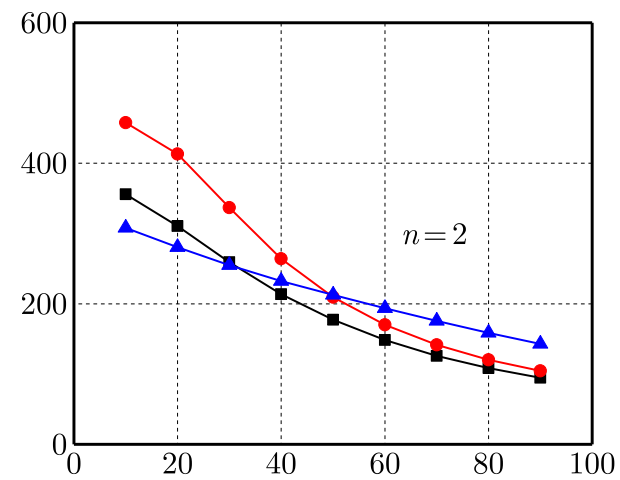

(f)

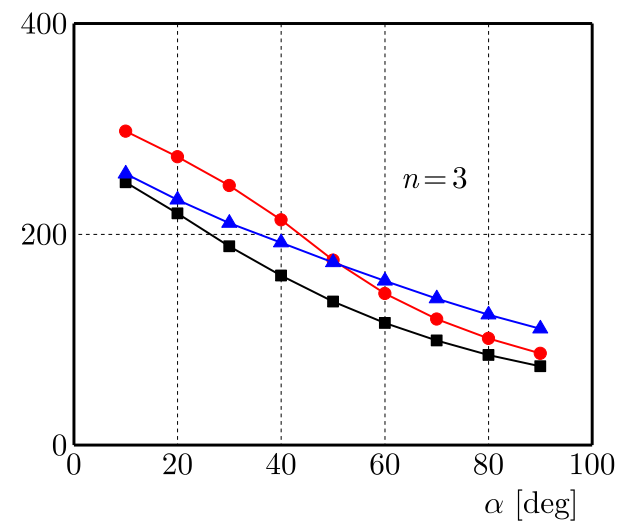

Fig. 8. The dependence of frequency of the mode- $z$, mode- $x$, and mode-tor investigated as a function of $\alpha$ for $n=1,2,3$ : (a)-(c) for Type 1 and (d)-(f) for Type 2

and opening angle $\alpha$ from $80^{\circ}-90^{\circ}$. The performance of the two types of serpentine springs are almost the same based on analyzing the mode coupling, however the device using the spring consisting of circular arcs is more compact than that using the spring consisting of straight beams. To obtain the operation mode to be the first mode, the number of turns and the opening angle of the spring should be modified in the increased trend.

\section{Acknowledgment}

This research is funded by the Hanoi University of Science and Technology (HUST) under project No. T2020-SAHEP-024. 


\section{References}

1. Barillaro G., Molfese A., Nannini A., Pieri F., 2005, Analysis simulation and relative performances of two kinds of serpentine springs, Journal of Micromechanics and Microengineering, 15, $4,736-746$

2. Chou H.M., Lin M.J., Chen R., 2016, Investigation of mechanics properties of an awl-shaped serpentine microspring for in-plane displacement with low spring constant-to-layout area, Journal of Micro/Nanolithography, MEMS, and MOEMS, 15, 3

3. Gu L., Li X., Bao H., Liu B., Wang Y., Liu M., Yang Z., Cheng B., 2006, Single wafer-processed nanopositioning XY-stages with trench-sidewall micromachining technology, Journal of Micromechanics and Microengineering, 16, 7, 1349-1357

4. Hieu D.V., Tam L.V., Duong N.V., Vy N.D., Hoang C.M., 2020, Design and simulation analysis of a $\mathrm{z}$ axis microactuator with low mode cross-talk, Journal of Mechanics, 36, 881-888

5. Hieu D.V., Tam L.V., Hane K., Hoang C.M., 2020, Design and simulation analysis of an integrated XYZ micro-stage for controlling displacement of scanning probe, Journal of Theoretical and Applied Mechanics, 59, 143-156

6. Hongwen L., 2004, Mechanics of materials, Higher Education Press, 201010, 5, 87-119

7. Huang Y.J., Chang T.L., Chou H.P., 2009, Novel concept design for complementary metal oxide semiconductor capacitive Z-direction accelerometer, Japanese Journal of Applied Physics, 48,7

8. Legtenberg R., Groeneveld A.W., Elwenspoek M., 1996, Comb-drive actuators for large displacements, Journal of Micromechanics and Microengineering, 6, 3, 320-329

9. LiU X., Kim K., Sun Y., 2007, A MEMS stage for 3-axis nanopositioning, Journal of Micromechanics and Microengineering, 17, 9, 1796-1802

10. Lobontiu N., Garcia E., 2005, Mechanics of Microelectromechanical Systems, Kluwer Academic Publishers, 167-178

11. Matsumoto Y., Nishimura M., Matsuura M., Ishida M., 1999, Three-axis SOI capacitive accelerometer with PLL C-V converter, Sensors and Actuators A: Physical, 75, 1, 77-85

12. Nguren M., Ha N., Nguyen L., Chu H., Vu H., 2017, Z-axis micromachined tuning fork gyroscope with low air damping, Micromachines, 8, 2, 42

13. Peroulis D., Pacheco S.P., Sarabandi K., Katehi L.P.B., 2003, Electromechanical considerations in developing low-voltage RF MEMS switches, IEEE Transactions on Microwave Theory and Techniques, 51, 259-270

14. Rouabah H.A., Gollasch C.O., Kraft M., 2005, Design optimisation of an electrostatic MEMS actuator with low spring constant for an "atom chip", NSTI-Nanotech, 3, 489-492

15. Sharaf A., SEDKy S., 2012, Design and simulation of a high-performance Z-axis SOI-MEMS accelerometer, Microsystem Technologies, 19, 8, 1153-1163

16. Su G.-D.J., Hung S.H., Jia D., Jiang F., 2005, Serpentine spring corner designs for micro-electro-mechanical systems optical switches with large mirror mass, Optical Review, 12, 4, 339-344

17. Weinberg M., Kourepenis A., 2006, Error sources in in-plane silicon tuning-fork MEMS gyroscopes, Journal of Microelectromechanical Systems, 15, 3, 479-491 